\title{
Kinematic Design, Analysis and Simulation of a Hybrid Robot with Terrain and Aerial Locomotion Capability
}

\author{
Sreevishnu S. ${ }^{1, *}$, Monish Koshy ${ }^{1}$, Anjai Krishnan ${ }^{1}$ and Gautham P. Das ${ }^{2}$ \\ ${ }^{1}$ Department of Mechanical Engineering, Amrita Vishwa Vidyapeetham, Amritapuri, India \\ ${ }^{2}$ Lincoln Centre for Autonomous Systems, School of Computer Science, University of Lincoln, United Kingdom
}

\begin{abstract}
Having only one type of locomotion mechanism limits the stability and locomotion capability of a mobile robot on irregular terrain surfaces. One of the possible solution to this is combining more than one locomotion mechanisms in the robot. In this paper, robotic platform composed of a quadruped module for terrain locomotion and quadrotor module for aerial locomotion is introduced. This design is inspired by the way which birds are using their wings and legs for stability in slopped and uneven surfaces. The main idea is to combine the two systems in such a way that the strengths of both subsystems are used, and the weakness of the either systems are covered. The ability of the robot to reach the target position quickly and to avoid large terrestrial obstacles by flying expands its application in various areas of search and rescue. The same platform can be used for detailed 3D mapping and aerial mapping which are very helpful in rescue operations. In particular, this paper presents kinematic design, analysis and simulation of such a robotic system. Simulation and verification of results are done using MATLAB.
\end{abstract}

Keywords- Hybrid mobile robot, search and rescue, legged flying robot

\section{Introduction}

Robots are very useful in places where human intervention is dangerous or impossible. Application of drones for search and rescue [1], volcano environment monitoring [2] and military operations are some examples for this. Using only one type of locomotion is not enough in this type of scenarios. One way of solving this issue is by using collaborative multi-robotic systems $[3,4]$. Another approach is using robot with hybrid locomotion capabilities [5]. The hybrid mobile robotics expanded the application horizon of robots to challenging areas in which multiple modes of mobility are required. This paper mainly focuses on development of a robotic platform for rescue operation and its possible applications in rescue fields.

Even though, polymorphic tracked vehicles are the state of art in terrain locomotion of rescue robots [6-8], researches are boosting in legged robotics field too [4]. Terrain robots can be used to create detailed 3D map of the environment, which would be very useful for rescue operators to find people underneath things. However, large obstacles and pits are difficult to surpass for terrain robots with legs or belt drive. Recent advent of drone technology had given a big propulsion to rescue robotics. Drones are used for 3D mapping and can be sent in to areas where it is too dangerous for human [2]. One big advantage with drones is that they are first responders.
Also, it can tackle big obstacles and pits in the rescue field. On the other hand, navigation of drones through narrow paths like caves and tunnels is difficult. Also, for doing detailed 3D mapping terrain close flying is required. This is difficult with drones in cluttered disaster sites.

To tackle these scenarios, the robot should be able to reach the spot as quick as possible and have to get into the field to analyse the situation. It is in this context the "Hybrid Locomotion Mobile Robot (HyLMoR)" is introduced. It is a hybrid locomotion mobile robot with flying and terrain locomotion capabilities. In particular, this paper describes an experimental study of combining aerial and terrain locomotion capabilities in a single robot with its kinematic study and simulation.

This paper briefly describes an experimental study of combining aerial and terrain locomotion capabilities in a single robot. Section II discusses work done in this area till now. Kinematic analysis, design and verification of quadruped module and quadrotor modules are presented in Section III and Section IV respectively. Then, these modules are combined to form HyLMoR, which is presented in Section V. Finally, the conclusion and future works are discussed. 


\section{Related works}

Concept of hybrid locomotion mobile robotics system started in early 1980s [9]. Combination of different terrain locomotion mechanisms constitutes hybrid systems in those days. Later researches combined different environmental locomotion capabilities like aquatic-terrain [10], aerial-terrain [5, 11, 12], aerialaquatic [13] etc.

Research on combining aerial and terrain location capabilities in a single robot is still in its infancy stage. At the initial stages of development, wings were used to achieve aerial capability. Morphing Micro Air-Land Vehicle (MMALV) [22] was one among first in this category. The design used in this robot is similar to that of insects. It uses wings for aerial motion and wheel-legs for terrestrial motion. Thus, long distance travel is possible with flight mechanism, while crawling mechanism helps in close inspection and surveillance. However, the winged aerial-terrain hybrid robots have many disadvantages such as lack of efficient controllability, inefficient transition from flight mode to terrain mode and vice versa and turbulence caused by variations in air speed from one wing to another [22].

Most of the drawbacks with winged flight are resolved with introduction of rotary flight technology. A robot developed by Kossett et al. in 2009 with flight and wheeled locomotion capability, using propeller for flight motion, is one of the first among this category [14]. This offered the best characteristics of both helicopters and terrain vehicles.

Rotary flying technology opened a new domain in aerial robotic systems. DUCK robot [12], flying hexapod [15], HyTAQ [16] and Pens-FlyCrawl [5] are some of the robots which uses rotary flights. The robot which is presented in this paper have more similarity with flying hexapod [15]. Main differences with this robot are HyLMoR comprises of quadruped instead of hexapod. Another big difference is in the modularity of components. In HyLMoR, quadruped and quadrotor modules are combined as separate entities. This makes controllability easier and gives independence between modules. Similarly, the use of legged locomotion helps the HyLMoR to tackle obstacles on ground during terrain locomotion in comparison with the wheeled locomotion in HyTAQ and with the crawling locomotion in Pens-FlyCrawl.

\section{Quadruped module}

The process of implementing kinematic designing and simulation of the quadruped module is described here. First, analysis of single leg is done and verified using MATLAB robotics toolbox [20]. The 3D model created in MATLAB environment is used to verify the forward and inverse kinematics of the designed robot.

\subsection{Selection of legged robot}

The proposed robotic system consists of a terrain locomotion part and flying part. For terrain locomotion on irregular surface, the robot needs good stability compared to wheeled locomotion, therefore legged locomotion is selected. Number of legs and configuration of the legs are the two main factors which affects the stability and locomotion of robot.

Thus, the desired robotic platform can be developed by considering the following factors:

- Lowering number of legs reduces the turning area and gives more simplicity to driving mechanism [18].

- Statically stable walking requires atleast four legs [18].

- In the proposed design, legs of the quadruped part are chosen to have 3 DOF in order to decouple body motion with terrain irregularities [18].

Considering the above factors and cost (lesser number of actuators needed), 3 DOF quadruped system for terrain motion is selected.

\subsection{Conceptual design of leg}

Leg geometry is one of the most significant aspect of design, since it influences the overall performance of the system. Two main categories of leg configuration [19] are horse type and insect type. In horse type, knee joint is always situated under hip joint as in dog, horse and most other terrestrial mammals. In insect type, knee joint is positioned parallel or above the hip joint.

There are many advantages for insect configuration over horse type configuration especially on uneven terrains $[18,19]$. The arrangement of two corresponding hip joints in insect type has the convenience of positioning two actuators inside or very near to body. This helps in reducing the load over foot joint while foot placing. The arrangement also simplifies the leg kinematics. Another important feature which is very helpful in rescue scenarios is flexibility in leg movement. i.e. the leg can extend within a wide range in forward and backward, and upward and downwards. Compared to horse type model it is more energy efficient in most of the scenarios.

The HyLMoR is mainly targeted to be operated in rescue scenarios which have highly uneven terrains. Terrain locomotion will be better if the legs are more flexible. Energy efficiency is also an important factor for long rescue operations. Considering all these factors, insect type leg configuration is selected.

\subsection{Kinematic analysis of leg}

Kinematic analysis of a robot is the basic requirement of space planning, motion planning motion control and optimal design. There are two types of kinematic problems, Direct Kinematics and Inverse Kinematics. Both of them are analysed and verified for the proposed quadruped module using MATLAB.

Assigning frames is the first task while modelling a robotic system. Cartesian co-ordinate system is used here. Quadruped is a rigid body system with a centre body and four leg modules. Centre of body can be considered as the origin of the robot frame in which $\mathrm{Z}$ axis is pointing upwards, $\mathrm{Y}$ axis towards left and $\mathrm{X}$ axis towards front. Each leg is attached to the corner of 
rectangular robot body. These points are origins of leg base frames.

Co-ordinate frames of legs are assigned using Denvait Hartenberg (DH) notation. Each leg is made up of links and joints. DH notation can give the analytical description of spatial geometry of a leg with pose of each links relative to the leg base frame. The relation between the joint-variables and the pose of the foot gives the kinematic model of leg. $\mathrm{DH}$ representation of a quadruped leg is shown in Fig. 1 and parameters are shown in Table 1.

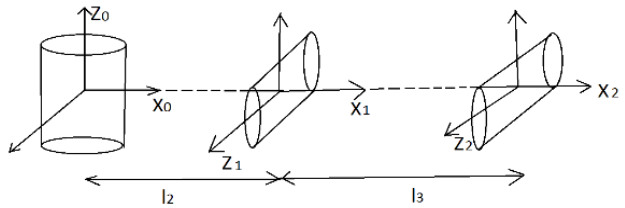

Fig. 1. DH representation of 3 DOF insect leg

Table 1. DH parameters of leg

\begin{tabular}{|c|c|c|c|c|}
\hline Link & $\theta_{\mathrm{i}}$ & $\mathrm{d}_{\mathrm{i}}$ & $\mathrm{a}_{\mathrm{i}}$ & $\alpha_{\mathrm{i}}$ (degree) \\
\hline 1 & $\theta_{1}$ & 0 & $\mathrm{~L}_{1}$ & 90 \\
\hline 2 & $\theta_{2}$ & 0 & $\mathrm{~L}_{2}$ & 0 \\
\hline 3 & $\theta_{3}$ & 0 & $\mathrm{~L}_{3}$ & 0 \\
\hline
\end{tabular}

Here, $\theta_{i}$ is the joint angle and $d_{i}$ is the joint distance. Parameters $a_{i}$ and $\alpha_{i}$ corresponds to link length and link twist.

Table 2. Link lengths taken for analysis

\begin{tabular}{|c|l|l|l|}
\hline Link & Link1 & Link2 & Link3 \\
\hline Length $(\mathrm{m})$ & 0.063 & 0.082 & 0.1536 \\
\hline
\end{tabular}

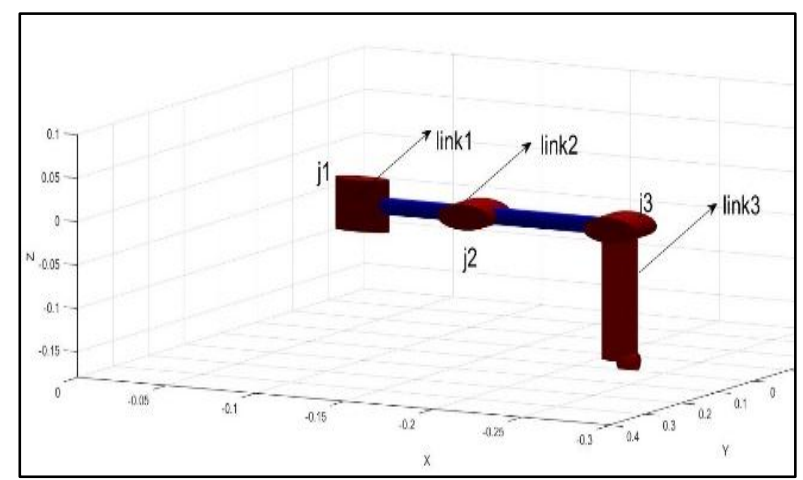

Fig. 2. Graphical representation of single leg

Link length is one of the important kinematic parameters. Length of the upper segment of the leg should be smaller than that of lower segment [21]. To start with, link lengths are selected in such a way that ratio of link lengths as 0.42 [21]. After considering mechanical constraints such as material property, leg configuration, etc., link lengths are adjusted slightly. Finally chosen link lengths for analysis are shown in Table 2. In Fig. 2, the 3D model of single leg is shown. Link1 corresponds to coxa, link2 corresponds to femur and link 3 corresponds to tibia respectively. Legs are attached to the corners of the rectangular body of dimension $20 \mathrm{~cm} \times 20 \mathrm{~cm}$. i.e., legs are attached radially at a distance of $14.14 \mathrm{~cm}$ from the centre of body. In the Fig. 2 joints are represented with j1, j2 and j3.

\subsubsection{Direct Kinematics}

Direct kinematics problem is finding the pose of leg tip pose as a function of the joint angle values. Homogeneous transformation matrices are used to find the leg tip pose with respect to leg base frame.

The individual joint transformation matrices formed by referring parameters in Table 1 . Are given in equations (1-3).

$$
\begin{aligned}
{ }^{0} T_{1} & =\left(\begin{array}{cccc}
c \theta_{1} & 0 & s \theta_{1} & l_{1} c \theta_{1} \\
s \theta_{1} & 0 & -c \theta_{1} & -l_{1} s \theta_{1} \\
0 & 1 & 0 & 0 \\
0 & 0 & 0 & 1
\end{array}\right) \#(1) \\
{ }^{1} T_{2} & =\left(\begin{array}{cccc}
c \theta_{2} & -s \theta_{2} & 0 & l_{2} c \theta_{2} \\
s \theta_{2} & c \theta_{2} & 0 & l_{2} s \theta_{2} \\
0 & 0 & 1 & 0 \\
0 & 0 & 0 & 1
\end{array}\right) \#(2) \\
{ }^{2} T_{3} & =\left(\begin{array}{cccc}
c \theta_{3} & -s \theta_{3} & 0 & l_{3} c \theta_{3} \\
s \theta_{3} & c \theta_{3} & 0 & l_{3} s \theta_{3} \\
0 & 0 & 1 & 0 \\
0 & 0 & 0 & 1
\end{array}\right) \#(3)
\end{aligned}
$$

Equations (1-3) can be concatenated to form homogeneous transformation matrix which gives the pose of foot of leg relative to base frame of leg. It is given by

$$
\begin{gathered}
{ }^{0} T_{3}={ }^{0} T_{1}{ }^{1} T_{2}{ }^{2} T_{3} \#(4) \\
{ }^{0} T_{3}= \\
\left(\begin{array}{cccc}
c \theta_{1} c\left(\theta_{2}+\theta_{3}\right) & -c \theta_{1} s\left(\theta_{2}+\theta_{3}\right) & s \theta_{1} & p_{x} \\
c \theta_{1} c\left(\theta_{2}+\theta_{3}\right) & c \theta_{1} c\left(\theta_{2}+\theta_{3}\right) & -c \theta_{1} & p_{y} \\
s\left(\theta_{2}+\theta_{3}\right) & c\left(\theta_{2}+\theta_{3}\right) & 1 & p_{z} \\
0 & 0 & 0 & 1
\end{array}\right) \#(5)
\end{gathered}
$$

where $c \theta_{i}$ is the cosine and $s \theta_{i}$ is the sine of $\theta_{i}$, for $i=1,2,3$ and

$$
\begin{aligned}
p_{x}= & l_{1}+\left(l_{2} c \theta_{2}+l_{3} c\left(\theta_{2}+\theta_{3}\right)\right) c \theta_{1} \#(6) \# \\
p_{y}= & l_{1}+\left(l_{2} c \theta_{2}+l_{3} c\left(\theta_{2}+\theta_{3}\right)\right) s \theta_{1} \#(7) \# \\
& p_{z}=l_{2} s \theta_{2}+l_{3} s\left(\theta_{2}+\theta_{3}\right) \#(8) \#
\end{aligned}
$$

The transformation matrix was verified for various known angle values, which result in known leg tip poses.

Transformation matrix at $\left(\theta_{1}, \theta_{2}, \theta_{3}\right)=(0,0,0)$ is given by

$$
{ }^{0} T_{3}=\left(\begin{array}{cccc}
1 & 0 & 0 & 0.2986 \\
0 & 0 & -1 & 0 \\
0 & 1 & 0 & 0 \\
0 & 0 & 0 & 1
\end{array}\right) \#(9)
$$

Transformation matrix at $\left(\theta_{1}, \theta_{2}, \theta_{3}\right)=(30,0,90)$ is given by 


$$
{ }^{0} T_{3}=\left(\begin{array}{cccc}
1 & -0.866 & 0.5 & 0.1256 \\
0 & -0.5 & -0.866 & 0.0725 \\
1 & 0 & 0 & 0.1536 \\
0 & 0 & 0 & 1
\end{array}\right) \#(10)
$$

\subsubsection{Inverse Kinematics}

The problem of finding the joint angles by using the leg tip pose is inverse kinematics. Let the pose of leg tip is given by transformation matrix,

$$
P=\left(\begin{array}{llll}
p_{11} & p_{12} & p_{13} & p_{14} \\
p_{21} & p_{22} & p_{23} & p_{34} \\
p_{31} & p_{32} & p_{34} & p_{35} \\
p_{41} & p_{42} & p_{43} & p_{44}
\end{array}\right) \#(11)
$$

Then, the joint angles $\theta_{1}, \theta_{2}$ and $\theta_{3}$ are given by

$$
\begin{gathered}
\theta_{1}=\tan ^{-1}\left(\frac{p_{24}}{p_{14}}\right) \#(12) \\
\theta_{3}=\cos ^{-1}\left(\frac{l_{3} p_{11}+p_{14}+l_{3} p_{21}+p_{24}}{l_{2}+l_{1}}\right) \#( \\
\theta_{2}=\theta_{3}-\cos ^{-1}\left(\frac{l_{3} p_{11}-l_{2} p_{21}+p_{24}}{l_{2}}\right) \#(
\end{gathered}
$$

\subsection{Leg trajectory generation}

To make the quadruped walking we need to generate a trajectory for each leg to follow. The tip of all the legs should move backwards at the same speed makes the body moving. The slippage at feet is assumed to be zero. Tripod gait is used here. In tripod gait, out of the four legs, at least any three of them will be touching the ground plane, with the perpendicular from the centre of mass falling within the triangle formed by the contact points of these legs. This gives stability to the robot while walking. Also, movement of each leg is limited with a small range such that it should not go too backward and too forward. Too much of forward or backward movements can cause collision between adjacent legs. However, a drawback of this approach is the large leg cycles needed for the robot motion.

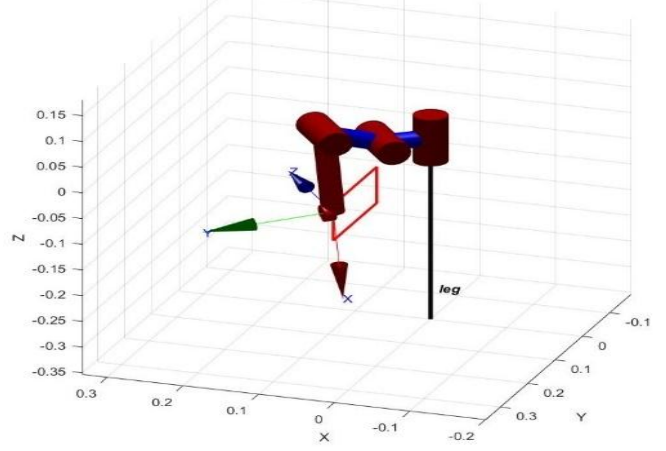

Fig. 3 . Snapshot from trajectory following of single leg
Considering all the above constraints, a rectangular trajectory is generated using the Robotic Toolbox function. For that, four via points are selected to generate the trajectory. Since there are four legs, a leg should be touching the ground for $3 / 4$ of full cycle and resetting time is only $1 / 4$ of full cycle. So, at the resetting phase, it should move faster. Rectangular trajectory followed by single leg is shown in Fig. 3. Since the legs are symmetric, the same trajectory with transformations can be used for other legs.

\section{Quadrotor module}

Kinematic design and analysis of quadrotor module of HyLMoR is presented in this section. The quadrotor module of the HyLMoR is a rigid body frame with cross structure and equally spaced arrangement of four rotors at each corner. Its six degrees of freedom can be controlled only by controlling the angular velocity of the four rotors. This makes it an underactuated system [17]. So, the six degree of freedom motion is achieved by coupling translational and rotational motions.

\subsection{Selection of frame and configuration}

Conventionally there are mainly two types of body frame structures, H or Dead Cat Frame and Cross frame. The cross-frame structure has been chosen for the HyLMoR due to the symmetrical design and mass concentration at the centre of the frame. Other advantages of cross frame in comparison with $\mathrm{H}$ frame structure are:

- It is a symmetrical design and propellers are placed equidistantly from the centre.

- Mass is concentrated at the centre.

- Control forces are distributed equally.

On the other hand, $\mathrm{H}$ structure is suitable for placing camera for wide view. However, HyLMoR is less affected by this, as the camera can be placed in the quadruped module. Cross structure can be controlled in two configurations, plus configuration and cross configuration. Usually while designing a quadrotor, configuration may not be considered since it is more related to control. However, here the quadrotor module can be placed over the quadruped module in these two configurations. So, selection of configuration is required at the time of designing itself. The following are some of the advantages of cross configuration over plus configuration which make it favourable to HyLMoR. -It gives more compactness to whole robot structure. -Robot will be more responsive in this configuration, since thrust is provided by two motors instead of one in the plus configuration.

-It produces more rotational acceleration, since effective distance from the centre is lower.

Analysing all these factors, cross configuration was chosen for HyLMoR. Now the placement of quadrotor over quadruped will be like quadrotor arms will be pointed to the direction of corners of quadruped. 


\subsection{Kinematics of quadrotor module}

The basic working principle of quadrotor is variable torques and thrusts. It consists of four rotor propellers each of these rotors actually results in certain flow of air and in turn a thrust onto the body of the quadrotor. Thus, firing up of four motors at the required speed provides rotational and translational motion to the quadrotor body.

To keep a position, thrust must be compensated for earth gravity and torques of all four rotors sum to zero. By varying velocities of the rotors without rotational moment, will results in altitude control of quadrotor module. Yaw motion can be obtained by applying nonzero net torque to the system by adjusting the rotor velocities. To move laterally, the thrust generated by lateral pairs of rotors should be different.

Kinematic modelling of quadrotor is done by assuming quadrotor as a symmetrical rigid body with centre of mass aligned with centre of body frame of the robot. The thrust provided by each rotor is proportional to the square of the angular speed. Quadrotor kinematics can be described using four reference frames [18]. An inertial frame $\mathrm{A}$, two intermediate frames, $\mathrm{E}$ and $\mathrm{F}$ and finally a body frame B. The inertial frame can be described with three orthogonal axis $a_{1}, a_{2}$ and $a_{3}$. The first intermediate frame $\mathrm{E}$ is formed with corresponding axis $e_{1}, e_{2}, e_{3}$ by rotating $A$ with respect to $a_{3}$ which is pointing upwards, by an angle $\psi$. The second intermediate frame $\mathrm{F}$ with corresponding axes $\mathrm{f}_{1}, \mathrm{f}_{2}$ and $\mathrm{f}_{3}$ is formed by rotating $E$ with respect to e 1 axis by an angle $\varphi$. Final body frame B with corresponding axes $b_{1}$, $b_{2}$ and $b_{3}$ is formed by rotating $F$ with respect to $f_{2}$ axis by an angle $\theta$. Fig. 4 shows frame assignment of quadrotor.

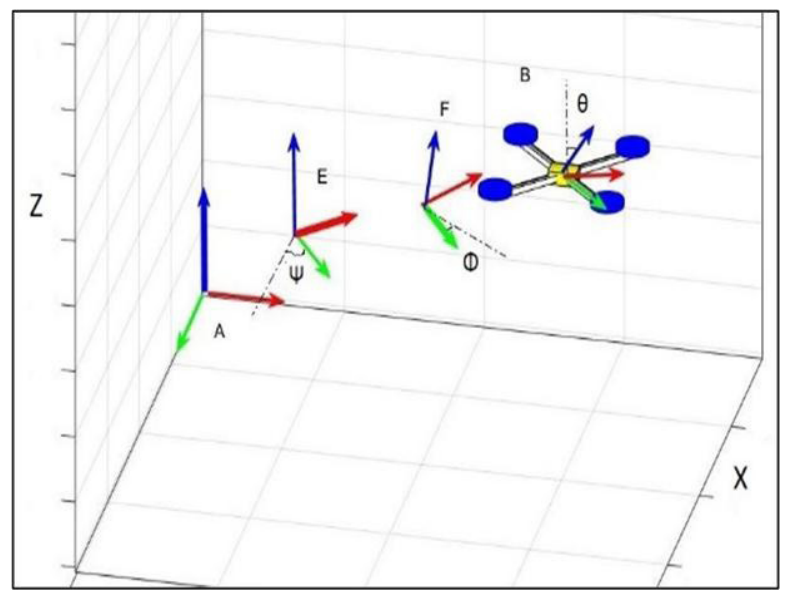

Fig. 4. Body fixed frame and inertial frame of quadrotor

The transformation between global frame A and body frame B is given as described below.

$$
X^{A}=R_{B} X^{B} \#(15)
$$

where ${ }^{A} R_{B}$ is the rotation matrix of frame $\mathrm{B}$ with respect to to frame $A$ and is given by

$$
{ }^{A} R_{B}=R(\psi) R(\phi) R(\theta) \#(16)
$$

where $R(\psi), R(\phi)$ and $R(\theta)$ are rotation matrices, given by

$$
\begin{aligned}
R(\psi) & =\left(\begin{array}{ccc}
c \psi & s \psi & 0 \\
-s \psi & c \psi & 0 \\
0 & 0 & 1
\end{array}\right) \#(17) \\
R(\phi) & =\left(\begin{array}{ccc}
1 & 0 & 0 \\
0 & c \phi & s \phi \\
0 & -s \phi & c \phi
\end{array}\right) \#(18) \\
R(\theta) & =\left(\begin{array}{ccc}
c \theta & 0 & -s \theta \\
0 & 1 & 0 \\
s \theta & 0 & c \theta
\end{array}\right) \#(19)
\end{aligned}
$$

Thus, from equations (17-19),

$$
\left.\begin{array}{ccc}
{ }^{A} R_{B}= \\
c \phi c \theta-s \phi s \psi s \theta & -c \phi s \psi & c \psi s \theta+c \theta s \phi s \psi \\
c \theta s \psi+c \phi s \phi s \theta & c \phi c \psi & s \psi s \theta-c \theta s \phi c \psi \\
-c \phi s \theta & s \phi & c \phi c \theta
\end{array}\right) \#(20)
$$

Results are analysed and verified at different known angles with known quadrotor poses. Rotation matrix of body frame $\mathrm{B}$ with respect to global coordinate frame A at angles $\psi=0, \varphi=0, \theta=0$ (See Fig. 5 . (a)),

$$
{ }^{A} R_{B}=\left(\begin{array}{lll}
1 & 0 & 0 \\
0 & 1 & 0 \\
0 & 0 & 1
\end{array}\right) \#(21)
$$

Rotation matrix of body frame B with respect to global co-ordinate frame As at angles $\psi=45, \varphi=0, \theta=0$ (See Fig. 5. (b)),

$$
{ }^{A} R_{B}=\left(\begin{array}{ccc}
0.7071 & -0.7071 & 0 \\
0.7071 & 0.7071 & 0 \\
0 & 0 & 1
\end{array}\right) \#(22)
$$

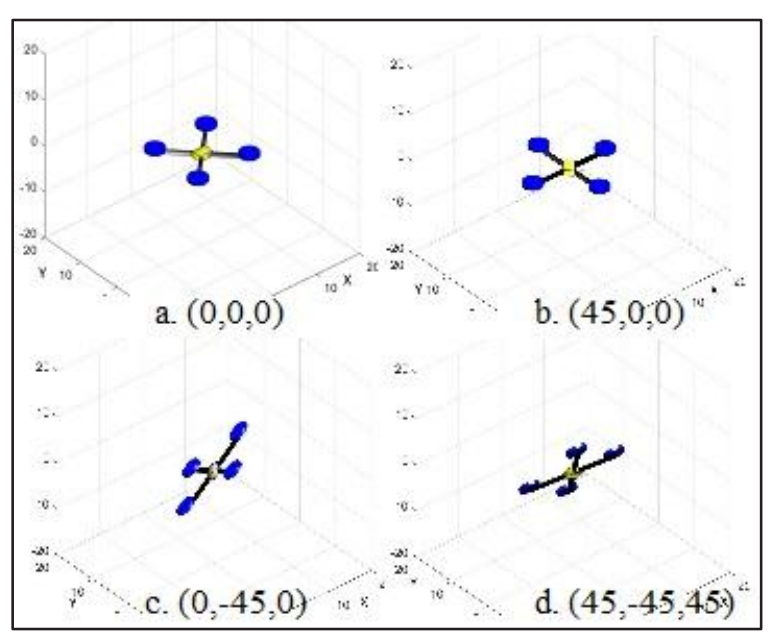

Fig. 5. Quadrotor pose at different angles $(\psi, \varphi, \theta)$

Rotation matrix of body frame B with respect to global co-ordinate frame $\mathrm{A}$ at $\psi=0, \varphi=0-45, \theta=0$ is given by (See Fig. 5. (c)), 


$$
{ }^{A} R_{B}=\left(\begin{array}{ccc}
0.7071 & -0.5 & -0.5 \\
0.7071 & 0.5 & 0.5 \\
0 & -0.7071 & 0.7071
\end{array}\right) \#(23)
$$

Similarly, at angles $\psi=45, \varphi=0-45, \theta=45$ (See Fig. 5 . (d)),

$$
{ }^{A} R_{B}=\left(\begin{array}{ccc}
0.8536 & -0.5 & 0.1464 \\
0.1464 & 0.7071 & 0.8536 \\
-0.5 & -0.7071 & 0.5
\end{array}\right) \#(24)
$$

\section{HyLMoR - Integration of modules}

As mentioned earlier, kinematic design of HyLMoR is done by combining quadruped module and quadrotor module. The two modules of the HyLMoR are analysed and verified separately. Now the robot can use its two locomotion modes to achieve a target position. One is walking mode and second one flight mode. Sometimes switching between modes is required to achieve a target position. Three snapshots from walking mode of simulation is shown in Fig. 6.

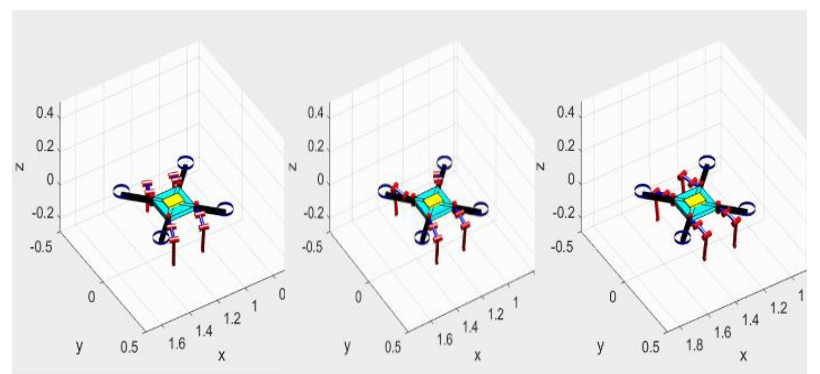

Fig. 6. Walking mode simulation at three continuous time frames

Simulation software mainly consists of quadruped and quadrotor kinematics and transformation updating and visualisation module. Simulation software collaboration diagram is shown in Fig. 7. The opensource robotic toolbox [20] of Corke is used for the development.

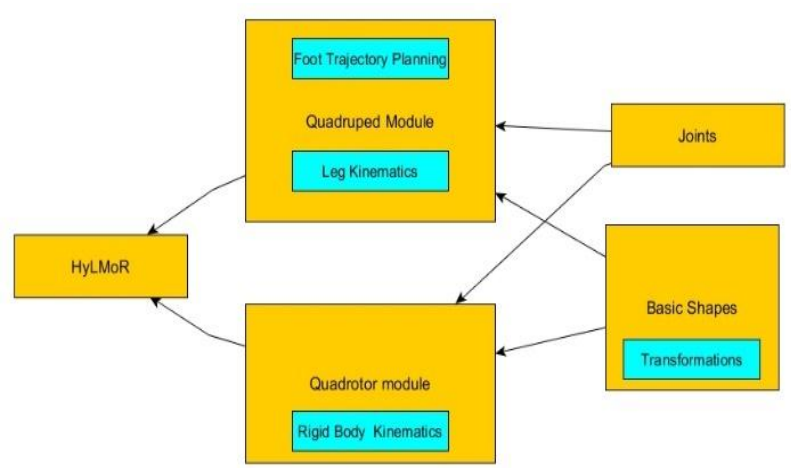

Fig. 7. Collaboration diagram of simulation software

\section{Conclusion}

In this paper, the project of experimental hybrid robot with aerial and terrain locomotion capability was briefly presented along with its application in search and rescue missions. Kinematic design, analysis and simulation was done to investigate the robot's ability to walk and fly. Analysis are done separately for each module. Later, two modules are combined in the simulation. Results are verified in MATLAB environment with multiple inputs. Leg motion was implemented using rectangular trajectory. Analysis and simulation results showed that the selected link lengths and leg spacings were good enough to avoid inter-leg collisions. Walking and flying simulations done for HyLMoR, resulted in proceeding the research further. The future studies include analysing the robot's dynamics, implementing gaits for moving on different difficult terrains and developing different behavioural controls for the robot.

\section{References}

1. D. Camara, Cavalry to the Rescue: Antibes Juan-les-Pins, (2014).

2. K. Nagatani et al., IEEE Int. Symp. Safety, Secur. Rescue Robot. SSRR, (2013).

3. Nathan Michael et al., Field and Service Robotics: Results of the 8th Int. Conf. , 92,33-47, (2013).

4. P. Fankhauser et al., IEEE/RSJ Int. Conf. Intell. Robot. Syst. IROS, (2016).

5. S. Kuswadi, M. N. Tamara, D. A. Sahanas, G. I. Islami, and S. Nugroho, Int. Conf. Knowl. Creat. Intell. Comput. KCIC, 80-87, (2016).

6. R. Murphy et al., Handbook of Robotics, 11621163,2008 .

7. J. Carlson and R. R. Murphy, IEEE Trans. Robot., 21, 423-437, (2005).

8. M. J. Micire, J. F. Robot., 25, 17-30, (2008).

9. Y. Ichikawa, N. Ozaki, and K. Sadakane, IEEE Trans. Syst. Man Cybern., (1983).

10. A. S. Boxerbaum, P. Werk, R. D. Quinn, and R. Vaidyanathan, IEEE/ASME Int. Conf. on Advanced Intell. Mechatronics, 1459-1464, (2005).

11. J. R. Page and P. E. I. Pounds, IEEE Int. Conf. Intell. Robot. Syst., 4834-4841, (2014).

12. C. J. Pratt and K. K. Leang, Proc. - IEEE Int. Conf. Robot. Autom., 3267-3274, (2016).

13. R. J. Lock, R. Vaidyanathan, and S. C. Burgess, Proc. IEEE RAS EMBS Int. Conf. Biomed. Robot. Biomechatronics, 681-687, (2012).

14. A. Kossett, J. Purvey, and N. Papanikolopoulos, IEEE/RSJ Int. Conf. Intell. Robot. Syst. IROS, 5653-5658, (2009).

15. M. C. Pitonyak, Syst. of Systems Engineering Conf. (SoSE), (2017). 
16. A. Kalantari and M. Spenko, Proc. - IEEE Int. Conf. Robot. Autom., 4445-4450, (2013).

17. K. P. Valavanis and G. J. Vachtsevanos, Handb. Unmanned Aer. Veh., 1-3022, (2015).

18. Kar, D. C., J. Robotic Syst., 671-686, (2003)

19. S. Hirose, Some considerations on a feasible walking mechanism as a terrain vehicle, 3rd CISM-IFToMM Int. Symp. on Theory and Practice of Robots and Manipulators , 357-375, (1978).

20. P. I. Corke, Robotics, Vision \& Control : Fundamental Algorithms in Matlab, Springer, (2011).

21. M. F. Silva, Quadruped robot optimization using a generic algorithm, 11th Int. Conf. on Climbing and Walking Robots and the Support Technologies for Mobile Machines, CLAWAR'11,11-26, (2011).

22. R. J. Bachmann, F. J. Boria, P. G. Ifju, R. D. Quinn, J. E. Kline, and R. Vaidyanathan, IEEE Int. Conf. Intell. Robot. Syst., 24-28, (2005). 